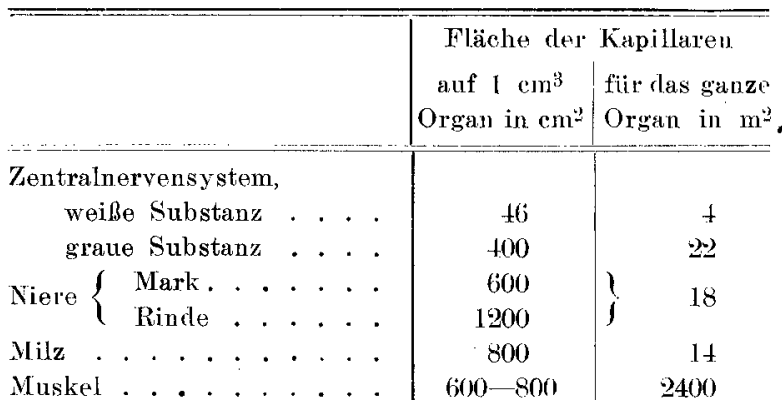

Die Gesamtfläche aller Kapillaren des groben Krrislaufs berechnet sich auf etwa $2900 \mathrm{~m}^{2}$, wovon allein $2400 \mathrm{~m}^{2}$ auf die Kapillaren der Muskulatur entfallen, dazu kommen noch etwa $1.40 \mathrm{~m}^{2}$ für: die Kapillaren des kleinen Kreislaufes, so daß beim Menschen die Fläche aller Kapillaren rund $3000 \mathrm{~m}^{2}$ beträgt. Diese große Fläche kann natürlich leicht große Flüssigkeitsmengen in das Blutgefäßsystem hinein oder aus ihm hinaus transportieren. So fand z. B. Magnus, daß etwa füuf Minuten, nachdem einem Hunde von $9 \mathrm{~kg}$ Gewicht $2260 \mathrm{~cm}^{3}$ verdünnte Kochsalzlösung ins Blut injiziert waren, sich im Blut nux noch $272 \mathrm{~cm}^{3}$ nachweisen ließen, rund $2000 \mathrm{~cm}^{3}$ waren in der kurzen Zeit durch die Kapillarwände hindurchgetreten. Man kann die Fläche der Kapillaren beim Hunde auf etwa $292 \mathrm{~m}^{2}$ schätzen, so daß der Flüssigkeitstransport pro $\mathrm{m}^{2}$ und Stunde $68,5 \mathrm{~cm}^{*}$ beträgt, ein Wert, der weit hinter dem zurïckbleibt, was die Schweibdrüsen zu leisten imstande sind. Durch die Wände der Blutkapillaren wird auch beim Menschen eine viel geringere Flüssigkeitsmenge transportiert als durch die Drüsenzellen der Schweibdrüsen, denn die Fläche der Kapillaren ïbertrifft jeno der Drüsenzellen etwa um das 3,5 fache. $\Lambda$ us (iner Reihe von Erfahrungen über den Flüssigkeitstransport in den verschiedenen (Yanen kann man für die mittlere Flüssigkeitsmenge, die pro Stuinde durch $1 \mathrm{~m}^{2}$ Kapillarwandfläche hindurch1 ritt, etwa folgende Werte angeben:

$$
\begin{aligned}
& \text { für die Speicheldrüsen . . 7,5-10,8 } \mathrm{cm}^{3} \\
& \text {, . SchweiBdrüsen . . 5, } 5, \text {, } \\
& \text {, "Nieronkapillaren . 4--6 , }
\end{aligned}
$$

Rechnen wir als Mittelwert $5 \mathrm{~cm}^{3}$, so würden durch die $3000 \mathrm{~m}^{2}$ der Kapillaren des qanzen Körpers pro Stunde 15 Liter hindurchtreten, d. h. in jeder Richtung 7,5 Liter. Eine wic auberordentIich gute Durchmischung der Körpersäfte hierdurch bewirkt wird, kann man sich vorstellen, wenn man bedenkt, daß die ganze Menge des Blutplasmas beim Menschen nur etwa 4 Liter beträgt, so daß also fast die doppelte Menge pro Stunde die Blutbahn verläßt, und eine ebenso große Menge aus der Gewebsflüssigkeit in das Blut übertritt. Hiermit ist aber noch durchaus nicht die Leistungsgrenze der Kapillaren erreicht, die vielmehr einen noch etwa 12-14mal intensiveren Flüssigkeitsaustausch ermöglichen würde.

Es besteht eine unerwartete Einheitlichkeit der Leistungen so verschiedener Organe, die die verschiedensten Stoffe im Sinne einer Resorption oder
Sokretion transportieren, sobald man die Leistungen mit der Größe der aktiven Flächen vergleirht.

Wenn der Typus der Speichcldrïsen $4-5 \mathrm{~cm}^{3}$ pro $\mathrm{m}^{2}$ und Stunde secerniert, der Schweildrüsentypus 12-19 $\mathrm{cm}^{3}$, so bedeutet das, dals sich auf der Zellfläche dieser Organe eine Sekretschicht von 4 bis $5 \mu$ bzw. 12-19 $\mu$ Höhe ansammeln wïrde. Da z. B. die Zellen des Pankreas etwa $17 \mu$ hoch sind, so wïrde die mittlere stündliche Sekretmenge nur eine Schicht von $1 / 3$ der Zellhöhe bedeuten, und selbst bei maximaler Sekretion würde die Dicke der pro Stunde gelieferten Sekretmenge noch nicht die Töhe der Zellschicht erreichen.

Selbst die größten sekretorischen Leistungen scheinen nicht mehr besonders erstaunlich, wenn man sich vergegenwärtigt, wie grol die Flächen sind, welcho sie vollbringen ${ }^{1}$ ).

\section{Die Brachiopoden des Kambriums von Nordamerika.}

Fon Dr. Cart T. Henning. Denuer Colo. U. S. A.

Die kambrische Periode ist das älteste geologische Zeitalter, aus dem uns zahlreiche Fossilien erhalten sind. Die meisten dieser Petrefakte stellen die Muscheln oder andere harte Teile mariner Lebewesen dar, die in den Sanden und Sedimentärablagerungen jener Zeit abgelagert wurden. Damit ist froilich nicht gesagt, daß im Kambrium überhaupt erst die ersten und einfachsten Lebewesen uns entgegentreten. Wir besitzen auch aus der vorkambrischen Zeit, dem Archaikum und Algonkium. für welch letzteres erdgeschichtliche Zeitalter in der amerikanischen Geologie das Wort ..Proteroatr: r.a" (nach dem Vorgange Thom. (C. Chamberlins) gebraucht wird, Ủberreste von Lebewesen, aber diese sind bisher noch nicht in genügender Zahl aufgefunden worden, um eine genaue systematische Reihenfolge der einzelnen Spezies aufzustellen. Erst mit dem Kambrium beginnt "reges Leben“, und gerado uach Nordamcrika müssen wir unsere Blicke wenden, wenn wir die vielgestaltige Tierwelt dieser Periode begreifen wollen.

Es ist das unbestrittene Verdienst Charles $D$. Walcots, dos früheren Direktors der U. S. Geological Survey und jetzigen Sekretärs der Smithsonian Institution in Washington, seit 30 Jahren sich fast unausgosetzt der Paläontologie des Kambriums gewidmet und als Bahnbrecher in der paläontologischen Forschung iberhaupt gewirkt zu haben. Abgesehen von seinen diesbezüglichen, grundlegenden Arbeiten in den Berichten des U. S. National Museums und der Smithsonian Institution, hat Walcott zum ersten Male im 10. Annual Report dex U. S. Geological Survey (1888/89) in seiner Abhandlung: ,F auna of the Lower Cambrian or Olenellus zone" (ibid. S. 509 bis 763, Taf. 43-98) die Paläontologie der Brachiopoden ausführlich behandelt, jener Gruppe von Tieren, die man fräher wegen ihrer äuBeren Ähn-

1.) Nähere Anqaben siehe A. Nüter, Aktive Ober fläche und Organfunltion. \%. f. allgem. Physiol. Bd. 12. 1910 , p. $195-214$. 
lichkeit mit Muscheln zu den. Weichtieren rechnete, heute aber als eine eigene Klasse betrachtet.

Kürzlich hat nun der Genannte die Wissenschaft mit einex umfangreichen Monographie bereichert, die wohl auf Jahrzehnte hinaus als das grundlegende Werk über' die „ArmfüBer" gelten wird:

Cambrian Brachiopoda. In \& Bänden. 1912. Teil I, 872 S.; Teil II, 104 Lichtdrucktafeln. Monograph 51 der U. S. Geological Survey. - Preis für beide Bände \& Doll., nach dem Ausland zuzüglich Porto.

Die Vorarbeiten zu diesem Werk begann Walcott unmittelbar nach Veröffentlichung der vorerwähnten Studie im 10. Ann. Rep. der Survey, doch haben Berufspflichten und andere Arbeiten den im 63. Lebensjahr stehenden Gelehrten verhindert, die große Monographie früher erscheinen zu lassen, an der er sich gleichzeitig der Mithilfe einer großen Anzahl amerikanischer und europäischer Forscher zu ertreuen hatte.

Die Monographie behandelt die über 1000 verschiedene Arten aufweisenden Brachiopoden historisch, geologisch und zoologisch. Es ist von selbst klar, daf man einem solch bedeutenden Werk nicht in einem Referat, auch wenn es noch so ausführlich wäro, gerecht werden kann. Der Zweok dieser kurzou Besprechung soll nur: der sein, das Werk auch bei den doutschen Frounden der Geologie und Paläontologie einzuführen.

In den einleitenden Kapiteln gibt Walcott zunäclast eine erschöpfende Bibliographie (S. 13-26), ein Referat über synonyme Ausdrücke (S. 27-98) und eine alphabetisch geordnete ṫbersicht über die geographische und stratigraphische Verteilung der Brachiopoden (S. 98-291), an die sich eine zoologische Terminologie anschliest (S. 291-296), wodurch auch der der Paläontologie ferner Stehende ein Verständnis für die zahlreichen technischen Ausdrücke, die in dieser Wissenschaft ebenso wenig fehlen wie in einer anderen, gewinnt. Hieran schließt sich die Einzelbeschreibung der unendlich vielseitigen 'Tiergruppe, die uns in dem die Tafeln enthaltenden Band lebenswahr entgegentritt. Tm eine allgemeine Übersicht über den Entwicklungsgang der einzehen Brachiopodenspezies zu gewinnen, hat $W$ alcolt ein provisorisches Diagramm entworfen, das die Entwicklung der Gruppe von den einfachsten bis zu den vollendetsten Formen anschaulich machen soll.

Bevor ich indessen den speziellen Teil des Werkes kurz skizziere, seien einige Bemerkungen vorausgeschickt.

Kambrische Schichten sind in Nordamerika weit verbreitet, ja man kann sagen, daß der nordamerikanische Kontinent das Kambrium in seiner größten Entfaltung zeigt. Während des Unteren Kambriums (Georgian-Stufe: Olenellas-Schichten) war nach den eingehenden Untersuchungen von Ulrich und Schuchert der ganze mittlere Teil des Kontinents trocken. Nur am Ost- und Westrande war Meer, und wird die Gleichmäßigheit der marinen Verhältnisse durch die Gleichförmigkeit der Fauna an den beiden Küstenlinien des Kontinents bestätigt. Des weiteren ist es eine gesicherte Tatsache der Geologie, daß die grolien Seebecken und Kontinentalmassen ihre relativen Lagen schon während des Algonkiums inne gehabt haben müissen. Im mittleren Kambrium (Acatian-oder St.- John-Stufe: Paradoxides undäquivalente Trilobitenschichten) kam vom Stillen Ozean und rom Süden her eine sich rasch ausbreitende Transpression, dic das ganze Innere des Kontinents bis weit nach Osten überflutete. Es entstand das sog. ,inneramerikanische" Meer oder, wie Walcott es nenut: die Mississippisee. „Das Meer drang langsam über das Land vor und verteilte die Zertrümmerungsprodukte, die von ihm verarbeitet oder von seinen Zuflüssen ihm zugeführt wurden, als Sandbänke längs der Küste. Von Arizona bis Texas, Missouri, den Black Hills, dem östlichen Rand der Rocky Mountains und läng's der Nordgrenze in Minnesota, Wisconsin, Michigan, Cauada und den Adirondacks von New York ist das Bild dasselbe: Sandstein, der auf vorkambrischen Gesteinen lagert und im wesentlichen dieselbe Fauna einschlie Bt. In einigen Gegenden nahm die Tiefe des Meeres rasch zu und kalkige Sedimente häuften sich auf dem Sande an, wie in Arizona, Texas, den Black Hills und an einigen Punkten am Ostfluß der Rocky Mountains" (Walcott). - Nur verhältnismäBjg kleine Teile des Nordostens der Vereinigten Staten und Canadas blieben rou der großen Transgression unberührt und hielten ihre Verbindung mit dem Atlantischen Ozean aufrecht. Im weiteren Verlaut des Mittleren Kambriums aber trat allmählich ein Zurückgehen des Mississippisees ejn, bis im Oberen Kambrium Potsdam-LSaratogan-IStufe; Dicellocephalus- oder Peltura-Schichten) cine weitere bedeutende Transgression erfolgte und das Meer noch weiter um sich griff. Die Potsdamserien (Santsteinc) bedecken nicht nur die östlichen und westlichen Randländer des Kontinents, sondern fiuden sich auch weit verbreitet im Innern. Das Obere Kambrium ruht im Osten und Westen konkordant auf den Schichten des Mittleren Kambriums, dagegen im Innern diskordant auf präkambrischen. Schichten.

Die ältesten kambrischen Fossilien finden sich in den Schichten des Unteren Kambriums des sülwestlichen Nevada und im benachbarten Tnyo CountyAreal des östlichen Kalifornien. In Abteilungen, die etwa 120 Meilen voneinander entfernt liegen, hat dort das Untere Kambrium eine Mächtigkeit von nahezu $2000 \mathrm{~m}$ bei einer aus Kalkstein bestehenden Deckschicht von 250 bis $700 \mathrm{~m}$. Unterhalb dieses Kalksteinlagers treten kalkhaltige Schichten auf, neben Sandstein, kieseligen und kalkigen Schiefern.

Nach Walcotl ist die primitivste, aber nicht älteste, Spezies aus dem Kambrium die Rustella edsoni. Sie findet sich vergesellschaftet mit Olenellus thompsoni in Vermont, wo sie vom obersten Unteren Kambrium bis zur Basis des kambrischen Systems vorkommt. Walcott vermutet, daB Tremotobolus excelsis der älteste Brachiopode ist; die Form ist bereits hoch entwickelt und muß deshalb eine lange Entwicklung aus den primitiven Brachiopoden (Protegulum-Stufe) durchlaufen haben. Rustella edsoni stellt gleichzeitig dic primitivste Form der Gattung Atremata dar. Neben ihr tritt 
Micromitra im Unteren Kambrium der kanadischen Rockies auf, welche Gattung sich bis zum Schlul. her Oberen Kreide erhält, um dann in Mickwitzia. die ebenfalls im Unteren Kambrium auftritt, und in Helmersenia und Volborthia des Oberen Kambriums überzugehen. Obolus wird etwas später als Micromitra bekannt und dauert bis in das Untere Silur, um sich dann in mehrere Subspezies zu teilen. Walcott ist der Ansicht, daB der Lingule lla ähnliche Formen aus der primitiven Obolus irr frühen Kambrium sich entwickelten, da die Art zuerst im unteren Teil des Unteren Kambriums bekannt wird und während des Verlanfs des gesamten kambrischen Systems bis ins Untere Silur fortlawert. Die Unterart Lingulepis erscheint im Mittleren Kambrium und erhält sich bis ins Untere Silur.

Dio Gattung Neotremata der Brachiopoden reieht vom Cnteren Kambrium bis ins Untere Silur und hat in Aerothele ihre alteste und einfachste Form, von der Acrotreta, und von dieser Acrothyra und Discinopsis sich ableiten. Ein anderer Zweig ist in Trematobulus vertreten, die wahrscheinlich der direkte Vorläufer der Siphonotretidue, einer Abzweigung der primitiven Obolella, ist. Yorlia tritt im Unteren Kambrium, Dearbornia im oberen Teil des Mittleren Kambriums auf.

Als Vorfahren der echten Protremata kommen. Nisusia und Billingsella in Betracht. Beide scheinen von jenen Stammformen zu kommon, die von Micromitna zu Kutorgina des Unteren Kambriums führt. Ein charakteristischer Vertreter der Gattung Protremata ist ferner Schuchertina, während Eoorthis erst in den unteren Schichten des MittJeren Kambriums auftritt und durch das Obere Kambrium bis in das Untere Silur vorkommt.

Bei dem Versuche, zwischen den Billingseliden des Kambriums und den Orthiden des Unteren Silur eine genetische Beziehung ausfindig zu machen, bieten die paläontologischen Reste insofern Schwierigkeiten, als eine große Lücke zwischen den Versteinerungen des Oberen Kambriums und des mittleren Unter-Silur die Vergleiche sehr erschwert. Die Orthiden des mittleren Unter-Silur schließen vine grobe Gruppe von Brachiopoden ein, die grundsätzlich von der Gruppe der Billingselliden in bezug auf den Bau der Schale verschieden sind. Daß diese Liicke in der systematischen Reihenfolge der einzelnen Arten im Verlauf des Fortschritts der paläontologischen Forschung ausgefüllt werden wird, ist durchaus nicht unwahrscheinlich, und es bedarf nur einer sorgfältigen Untersuchung auch der kambrischen Schichten außeramerikanischer Erdteile, um möglicherweise hier die Brücke zu schlagen.

Die Fundstätten erwähnt Walcott hei jeder Art auf das genäueste, so daß sich cin gutes Bild der Verteilung der Brachiopoden ïbor den Kontinent gewinnen läßt. Summarisch betrachtet, kommt Walcott dabei zu folgendem Ergebuis:

Die Olenellus-Fama bevölkert die O- und WSeite eines Kontinents, der in seiner allgemeinen Konfiguration Nordamerika, wio es heute ist, darstelll. Gonan gesprochen lobte diese Famna aber nichl anf der dem Ozean zugewandten Küste, son- dern auf den Küsten der Binnenmeere, Sunde, Meeresarme oder Tagunen, die die Zwischenräume zwischen den verschiedenen Landmassen einnahmen, die aus der teilweise vom Meer bedeckten kontinentalen Plattform östlich und westlich des zentralkontinentalen Areals emporragten. Auf der Ostseite erstreckte sich das erste Land östlich vom zentralen Teil des Kontinents von Alabama nordöstlich längs der Linie der gegenwärtigen Kette der Appalachen bis zu den Green Mountains von Vermont, diese einschließend. Ob die Fauna auch in der Gegend des Connecticut River und von da bis ostlich von den Green Mountains vorkam, ist nicht bekannt. Daß. sie weiter östlich vorkam, wird durch ihr Auftreten im östlichen Massachusetts und nordwestlichen Neufundland bewiesen. Noch weiter ostlich kommt sie auf der Halbinsel Avalon vor., bis zu der Stelle, wo die archäischen Gesteine das zentrale Neufundland kreuzen. Zur Stütze dieser Sätze stellt Walcott folgende Hauptsätze aut:

1. Die die Olenellus-Fauna enthaltenden Schichten sind. nur in den ostlichen und westlicluen Teilen des Kontinents bekannt;

2. soweit bis jetzt bekannt, fehlen Schichten des Unteren Kambriums in Innern des Kontinents;

3. die Schichten des Oberen Kambriums überlagern diskordant die (Yesteine des Algonkian und Archäen in jenen Provinzen, wo Schichten des Mittleren und Tnteren Kambriums fohlen;

4. die Schichten des Mittleren und Unteren Kambriums unterlagern konkordant jene des Oberen Kambriums auf der $O$ - und W-Seite des Kontinents ïberall da, wo die drei $\mathrm{Ab}$ teilungen des Systems vorkommen.

Die physischen Verhältnisse des späteren Unteren Kambriums hielten bis ins Mittlere Kambrium an und waren in der Hauptsache gefolgt von einem graduellen Einsinken des Landes, Erosion und von Oberflächenveränderungen im kontinentalen Areal südlich von den Großen Seen. In dem Male, wic dann weiter das Meer über diese Areale und über die dem Appalachen- und Cordillerensystem benachbarten Kü̈stengebiete sich ausbreitete, war dem wärmeren Leben Gelegenheit zu reicher Formentfaltung gegeben. So ist die Paradoxides-Fama der atluntischen Küste und die Olenoides-Fauma des Pacific nur eine Illustration für das vielgestaltige Leben jener Zeit und jener Räume.

Auf die so überaus wichtige Frage der Biogenesis der Brachiopoden geht Walcott in seiner großen Monographie nicht ein; dagegen hat er diesen Punkt vor mehreren Jahren (November 1908) auf der Naturforscherversammlung in Baltimore kurz berübrt, und ich rebe seime diesbezïglichen ÄuBerungen ${ }^{1}$ ) nach dem Wortaut seines Essays über "Evolution of early paleologir faunas in relation to their environment" wieder.

1) Zuerst erschiemen im Journal of geology 1908 dann abgedruckt in Outlines of geologic history with especial reference to North America von $B$. Willis 11 15. D. Salisbury. - The University of Claimgo Press. 1910. S. $28-37$. 
Dais das Milice (enviroment) des Mitheren Kambiums einer raseheren Entwidkung der Lebewesen günstiger war als das Untere und Obere Kambrium, wird Inreh die stratigraphisehe Verteilung der Brachiopoden bewiesen. In den auf gewisse Areale beschränkten Gewässern des Unteren Trambriums lassen sich die Brachiopoden der ganzen Welt in 20 Arten and 75 Spezies nachweisen, im Mittleren Kambrium dagegen sind es 31. bzw. 243 und im Oberen Kambrium 23 bzw. 137. Bei den I'rilobiten walten ähnliche Verhältnisse vor. Walcolt stellt dann folgende Hauptsätze auf:

1. Mehr oder weniger gleichmäßige und gïnstige, selbst warme klimatische Verhältnisse müssen geherrscht haben, um eine so grobe Verbreitung von fast identischen, korallenähnlichen Organismen, wie sie im Unteren Kambrium vorkommen und der großen Zabl von Individuen verschiedener Spezies von Trilobiten usw. zu erklären, die sich im Mittleren Kambrium finden;

2. die schnelle und ausgeprägte Entwicklung der Fauna des Mittleren Kambriums ist in der Hauptsache auf die Ausbreitung der kambrischen Meere und die dadurch bedingte Verschiebung der Küstenlinien und durch Veränderung der Wohnplätze der Tiere usw. zu erklären;

3. die Diversifiziermng der Fauna des Mittleren Tambriums wird in der Hauptsache auf eine rapide Entwicklung der provinzialen oder isolierten Faunen zuxückzuführen sein, die ihrerseits wieder durch das Zusammenbrechon der tremmonden Landbarren sich mit her woiter verbroitcten Fauna vermisehte;

4. dic frole Bewegung der Storungen in deu kambrischen Meeren mub für die kosmopolitische Fauma des Frih-Paläozoikums verantwortlich gemacht werden.

Auf die Frage, wie das erste Leben im Kambrium bzw. in der präkambrischen Zeit entstanden ist, geht Walcolt nicht ein, und er tut auch recht daran. Amerikanischerseits ist diese "Frage aller Fragen" chenso zu lösen versucht worden, wie bei dem Volke der Denker. Es hiebe aber den Raum dieser Skinze weit überschreiten. wenn ich die verschiedenen diesbezüglichen Theorien hier folgen lassen wärde, denn vorläufig und wohl auch noch auf lange hinaus - müssen wir uns wohl oder übel der Worte erinnern, die vor 2500 Jahren der alte Xenophanes seinen Volksgenossen zurief:

Selbst wer die volle Wahrheit uns enthüllte,

Nie wiird' er wissen, ob es Wahrheit ist,

Denn alles, was wir sagen, bleibt Vermutung.

\section{Die Physik auf der 85. Versammlung Deutscher Naturforscher und Ärzte in Wien, September 1913.}

\author{
Von Prof. Dr. Kart Scheel, Berlin-Dahlem.
}

(Schlus.)

Vierte Sitzung: Mittwoch, den 24. September 1913, vormitags, gemeinsam mit don Abteilungen fitr Chemi*

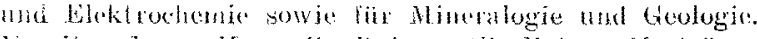
lorsitzenter; Herr H. Rubens (Berlin), Vorträge: 1. Herr 4 . t. Lathe (Zürich): "Uber Interfereng von Töngenstrahlen in Kristallen". Der Vortragende ent wirket cine Thorie der Interferenz der Röngenstrahen und bespridut larz die in wouerer $Z$ it anf diesem Gebiete unternommenon Versuche und Messungen. Sodann geht er auf die Bedeutum ger Röntgenstrableninterferenz fïr die Untexuchung der Struktur der Kristalle ein, insbesondere aut das Aussehen der Interferenzen bej Diamant sowie bei einer Reihe hemiedrischer Kristalle. Es gibt Fälle (Zinksultid, Diamant, Nickelsulfat), in melten kein Linflub der Hemiedrie za spüren ist. Dagegen zeigen Autuamen an Houentit und Pyrit, dab die Richtung parallel zur Würelkante keine vierzillige. sondern mur noch eine zweizhlige Achse dieser hemiedyschen Kristalle ist. Obwohl beide derselben Klasse angrehören, unterscheiden sich dennoch ihre Interferenzbilder. Auch die Schraubenstruktur des Quarzes, welche u. a. durch das optische Drehvermögen bewiesen wird, zeigt sich im Interferenzbild. Durchstrahlt man Recintsquarz parallel zur Hauptachse, so findet man keineswegs wie boim Beryll hexagonale symmetrie. Z.Wwar, richtet man seine Aufmerksamkeit allein auf dic Lage der Punkte, so bleibt diese Symmetrie bestchen; aber die Verteilung der Intensitaten entspricht iln nicht, so daß man tatsächlich nur dreizählige symmetric beobnchtet. Den Unterschied zwischen dem Reehts- and Linksquarz zeigen zwei Aufnahmen, bei welchn eine Nebenachse Durchstrahlungsrichtung war; die beiden Bilder gehen durch Spiegelung ineinander über. Stellt man die Frage ob es möglich ist. mit Hilfe der liöntgenstrahlinterferenzen die Struktur cines Kristalles vollstandig und eindentig zu bestimmen, so läßt sich diese nicht uneingeschrinkt bejalien. Wir stehen den Kristallen jetzt $\ddot{a}$ hnlich gegenïber, als sollten wir ein optisohes Gitter ohne Mikroskop, allein aus seinen Spoktren herans intersnchen. Die Gesamtheit dieser Spektren enthalt freilich, wie ja besonders 4 bbo betont hat, alle Wlemente, aus denen sich das mikroskopische Bild zasammensedzt. Aber zur Konstruktion dieses Mildes geniigt die Kenntnis der Lage und Intensitut der spektren nicht. Es kommt noch wesentlich auf die Phasen an, mit welchen diese gegeneinander schwingen. N.an miißte mindestens auch noch die Phasendifferenzen $z$ wischen den versehiedenen Interferenzpunkten eines Plotogramms feststellen; erst dann wäre es möglieh, zwingende Schlüsse auf Kristallstrukturen zu ziehen. 2. Herr H. Friellich (München): „Uber Röntgenstrahlinterferenzen". Jer vortragende behandelte im besonderen das Thema welche sehlusse sich aus den bisherigen Interferenzversudien mit Röntgenstrablen an Kristallen aut das Spektrum derselben sowie anf ihre Wellenlänge ziehen lassen. Sowohl die Interferenzerscheinungen, die beim Durchgang der Strahlen durch Kristalle beobachtet werden, wje diejenigen bei der Reflexion an Spaltflachen ergeben übereinstimmend das Vorhandensein eines kontinuierlichen Spektrums, das den durch das Kristallgitter als Dispersionsapparat zerlegten impuls darstellt und dessen Intensitätsmaximum mid Grenze nach den kurzen Wellenliugen hin von der Geschwindigkeit der die Rontgenstrahlen erzeugenden Kadhodenstrahlen abhüngig ist. Dieses kontinuierliche Spektrum wird uberlagert von einem zweiten linienartigen, dessen Hertunft die charakteristische Stellnng der Antikathode ist, das sich also für verschiedenes Material der Antikathode in bezug auf die lage und Intensität seiner Linien ändert. Die Größe der Wellenlänge der Röntgenstrahlen läßt sich wegen der nicht genügend eindeutig bestimmbaren Konstante der Kristallgitter absolut nicht ans diesen Trterferenzyer- 